Itinéraires Itinéraires

Littérature, textes, cultures

2011-1| 2011

Les Mémoires, une question de genre?

\title{
Art de dire et conception de la raison au siècle des Lumières
}

Marc André Bernier

\section{(2) OpenEdition}

1 Journals

Édition électronique

URL : http://journals.openedition.org/itineraires/1639

DOI : 10.4000/itineraires.1639

ISSN : 2427-920X

Éditeur

Pléiade

Édition imprimée

Date de publication : 1 avril 2011

Pagination : 95-105

ISBN : 978-2-296-13692-2

ISSN : $2100-1340$

Référence électronique

Marc André Bernier, "Art de dire et conception de la raison au siècle des Lumières », Itinéraires [En

ligne], 2011-1 | 2011, mis en ligne le 01 avril 2011, consulté le 26 juin 2020. URL : http://

journals.openedition.org/itineraires/1639; DOI : https://doi.org/10.4000/itineraires.1639

\section{(c) $(7)(9$}

Itinéraires est mis à disposition selon les termes de la licence Creative Commons Attribution - Pas d'Utilisation Commerciale - Pas de Modification 4.0 International. 


\title{
Art de dire et conception de la raison au siècle des Lumières
}

\begin{abstract}
In the late eighteenth century, Rivarol affirmed that "dry reason must yield to ornamented reason." This notion of ornamented reason deals with a figure of rationality that combines the charms of speech with the requirements of reasoning. Such, at least, is what we are invited to think by the works of the Marquise de Lambert, notably her Nouvelles réflexions sur les femmes (1727), a text that represents one of the most complete expressions of a culture dominated by an oratorical conception of reason.
\end{abstract}

Keywords : French Eighteenth Century, rhetoric, Enlightenment philosophy, women's studies, marquise de Lambert

Mots clés : dix-huitième siècle français, rhétorique, philosophie des Lumières, études féminines, marquise de Lambert

Une petite pièce anonyme tirée d'un numéro de 1742 des Amusemens du cour et de l'esprit résume sans doute au mieux la manière singulière dont le premier $\mathrm{XVIII}^{\mathrm{e}}$ siècle a envisagé les rapports entre la raison et l'éloquence :

La raison, come dit la Marquise de Lambert, doit plutôt regler les passions que les combattre, \& moins travailler au dessein chimérique de les déraciner de nous-mêmes, qu'à les assaisoner par le goût de l'esprit \& par les sentimens du cœur [...]. La raison a sa mollesse, \& sait plier aux choses qui conviennent à la nature d'une ame bien née $[\ldots]^{1}$.

À l'évidence, la sagesse que professe l'auteur de ce texte anonyme doit beaucoup à l'épicurisme antique, et peut-être davantage encore au libertinage érudit des Modernes qui s'en inspirent. C'est ce que montrerait

1. «Lettre sur les romans. A M**. Journaliste étranger. Ce premier septembre 1742 », Les amusemens du cour et de l'esprit, 1742, t. XIV, p. 420. 
sans peine la confrontation entre ce passage et quelques textes s'inscrivant dans le mouvement général de réhabilitation des passions et de promotion des plaisirs qui s'affirme au seuil des Lumières et au regard duquel, comme l'écrivait Nicolas Fréret dans sa célèbre Lettre de Thrasybule à Leucippe (vers 1720), « [n] ous naissons tellement disposés que nous recherchons le plaisir [...], et cette loi [...] est d'une telle autorité que nous ne pouvons nous empêcher d'y obéir $[\ldots]^{2} \gg$. Pourtant, pareille philosophie morale ne met pas seulement la raison à l'école des sens et du sentiment pour mieux l'inviter à se prêter aux exigences de la nature : elle lui confie encore le soin d' " assaisonner les passions par le goût de l'esprit » afin de conférer de la politesse aux plaisirs. Il en résulte un art de vivre appelé à s'épanouir dans un art de dire placé sous le règne de l'agrément, si bien que l'on ne saurait, dans ce contexte, envisager la raison sans les ornements et les assaisonnements que lui procure une parole aimable et éloquente. Cette conception oratoire d'une ratio s'accomplissant dans les grâces de l'expression s'exprime, au surplus, dans les rhétoriques du temps. Dans une Rhétorique françoise à l'usage des jeunes demoiselles qui paraît en 1745, un rhéteur mondain comme Gabriel Henri Gaillard n'assure-t-il pas que, sans les séductions de l'élocution, « les raisonnemens les plus solides, les mieux enchaînés, les mieux suivis, n’ont rien que d'ennuyeux \& de désagréable : sans elle, la raison même révolte ${ }^{3} »$ ?

À la fois rhétorique et philosophique, morale et stylistique, cette configuration complexe où s'entremêlent charmes de la parole et exigences du raisonnement se trouve résumée dans quelques formules emblématiques que reprennent et répètent à l'envi rhéteurs et écrivains du premier XVIII ${ }^{\mathrm{e}}$ siècle. Dans un cours de rhétorique professé au collège jésuite Louis-le-Grand de Paris en 1726, le père de La Sante parle ainsi de « raison assaisonné ${ }^{4} »$, locution qu'il tire par ailleurs d'une Épitre

2. Nicolas Fréret, Lettre de Thrasybule à Leucippe, éd. Sergio Landucci, Florence, Leo S. Olschki Editore, 1986, p. 323.

3. Gabriel Henri Gaillard, Rhétorique françoise à l'usage des jeunes demoiselles, avec des exemples tirés, pour la plupart, de nos meilleurs orateurs et poëtes modernes, seconde édition, revue, corrigée et augmentée, Paris, Huart et Moreau fils, 1748, p. 81. Sur cet ouvrage, qu'il s'agisse de ses principes de composition ou encore du public auquel il s'adresse, je me permets de renvoyer le lecteur à mon article, «Ad majorem feminarum gloriam. L'Essai de rhétorique à l'usage des jeunes demoiselles (1745) de Gabriel-Henri Gaillard et la tradition jésuite », dans Claude La Charité (dir.), Femmes, rhétorique et éloquence sous l'Ancien Régime, Saint-Étienne, Publication de l'Université de Saint-Étienne (sous presse). 4. Voir Gille Anne Xavier de La Sante, S. J., « Définition de l'esprit, par feu Mr. Rousseau », Rhetorica a patre dicta de la Sante anno millesimo septingentesimo vigesime septime in Collegio Ludovici Magni, Paris, 1727, Bibliothèque Sainte-Geneviève, ms. 3497, page de garde non numérotée : «Qu'est-ce que l'esprit? raison assaisonnée : / Par ce mot seul la dispute est donnée. / Qui dit esprit, dit sel de la Raison; / Donc sur deux points roule mon oraison. / Raison sans sel est fade nourriture : / Sel sans raison n'est solide pâture : / De 
à Marot du poète Jean-Baptiste Rousseau, lequel à son tour empruntait à Aristote cette métaphore de l'assaisonnement. Citons également une expression comme celle de "raison aimable », à laquelle recourt par exemple le cardinal de Bernis, lorsqu'il prétend que tous ses «écrits annonceront cette façon de penser, ou plutôt cette faculté de sentir » qui n'offre que « des tableaux rians [et] une raison aimable ${ }^{5}$ ». Suivant le même esprit, Rivarol affirme encore, à la fin du siècle, qu' " il faut que la sèche raison cède le pas à la raison ornée ${ }^{6} »$. Mais qu'il s'agisse d'une raison assaisonnée, aimable ou encore ornée, nous avons affaire dans tous les cas à une figure de la rationalité qui se place sous l'égide des arts de la conversation et du salon féminin. Voilà, du moins, ce qu'invite à penser l'œuvre de la marquise de Lambert et, notamment, ses Nouvelles réflexions sur les femmes, texte vraiment remarquable qui paraît en 1727 et sur lequel j'aimerais tout particulièrement attirer l'attention.

De fait, ces Nouvelles réflexions sur les femmes ont d'abord pour mérite de développer les principes définissant l'esprit qui régnait dans le salon qu'elle a elle-même tenu et où se réunissaient, tous les mardis, des gens de lettres comme Fontenelle ou Marivaux, l'abbé de Choisy ou le père Buffier, Madame de Tencin ou encore Madame Dacier. S'il fut assurément le plus brillant et le plus célèbre du premier tiers du $\mathrm{XVIII}^{\mathrm{e}}$ siècle, ce salon illustre surtout les aspects essentiels d'une pratique culturelle qui, comme l'écrit Voltaire, permet d' « apprendre ce que c'est que la raison ornée des grâces ${ }^{7} \gg$. Il représente même l'une des expressions les plus achevées de cette culture à laquelle préside l'idéal d'une raison ornée, comme en témoignent notamment ces deux vers qu'un certain abbé de Ponçol place en exergue du chapitre qu'il consacre à la marquise de Lambert dans un ouvrage paru sous le titre de Code de la raison, et qui se lisent comme suit :

Plaire, instruire à la fois, tel est son caractere
C'est Vénus au Lycée, ou Minerve à Cythere

Voilà, en somme, l'allégorie sous laquelle paraît cet art d'« assaisonner la raison » qui a dominé le premier $\mathrm{XVIII}^{\mathrm{e}}$ siècle et dont il reste maintenant à interroger les principes en examinant et en approfondissant tour à tour deux questions : d'une part, ce que j'appellerais volontiers, à

tous les deux se forme esprit parfait; / De l'un sans l'autre un monstre contrefait. » Cette définition est tirée d'une Épitre à Marot de Jean-Baptiste Rousseau.

5. François-Joachim de Pierre de Bernis, Euvres de François Joachim de Pierre, cardinal de Bernis, Paris, Didot, 1797, p. 241.

6. Antoine Rivarol, De l'universalité de la langue française, Paris, Cocheris, 1797, p. 31.

7. Voltaire, Voltaire à Ferney, sa correspondance avec la duchesse de Saxe-Gotha, éd. Évariste Bavoux et Alfonse François, Paris, Didier, 1860, p. 263.

8. Henri-Simon-Joseph Ansquer, abbé de Ponçol, Code de la raison, ou Principes de morale pour servir à l'instruction publique, Paris, Colas, 1778, t. II, p. 217. 
la suite de l'abbé de Ponçol, «Vénus au Lycée » ou l'idéal du salon féminin et, d'autre part, « Minerve à Cythère » ou comment la raison devient aimable.

\section{« Vénus au Lycée » ou l'idéal du salon féminin}

«Il y avoit autrefois des maisons où il étoit permis de parler \& de penser; où les Muses étoient en société avec les Graces ${ }^{9}$ », remarque la marquise de Lambert dans ses Nouvelles réflexions sur les femmes. Cet éloge du salon féminin sur lequel s'ouvre le texte appelle au moins trois commentaires.

Premièrement, la marquise de Lambert refuse d'emblée de dissocier art de parler et art de penser. Cet idéal est certes horatien, mais peutêtre surtout cicéronien. De fait, lorsque l'Art poétique rappelle à quel point il importe de joindre l'utilité à l'agrément (il s'agit de l'impératif classique de l'utile dulce) ou encore que le savoir et la sagesse sont les véritables sources de l'éloquence ("Scribendi recte sapere est et principium et fons $\left.{ }^{10} »\right)$, Horace ne fait, au fond, que mettre en maximes des principes qu'avait déjà énoncés et théorisés un ouvrage comme le $D e$ oratore de Cicéron. Texte où se manifeste au mieux, « suivant un sentiment assez général, la conception » que l'orateur latin « se fait de l'éloquence ${ }^{11} »$, le traité De l'orateur est, on le sait, conçu comme une suite de dialogues, dont le troisième est notamment consacré aux problèmes relatifs à l'élocution oratoire. Or, ce n'est pas tant la description technique des différentes figures de rhétorique qui importe alors, qu'une conception générale de l'art de dire qui se refuse à toute distinction de principe entre les res et les verba, c'est-à-dire entre le « fond » du discours et les « $\operatorname{mots}^{12} »$. «L'éloquence est une ${ }^{13}$ », écrit en effet Cicéron : elle est à la fois art de bien dire et art de bien penser ${ }^{14}$, et c'est à ce titre qu'elle confère au discours une force d'enchantement indissociable d'une alliance entre la parole et l'ensemble des savoirs que celle-ci met en œuvre. Une telle conception de l'ars dicendi renferme, de ce fait, une critique de la

9. Anne-Thérèse de Marguenat de Courcelles, marquise de Lambert, Nouvelles réflexions sur les femmes, dans Euvres de Madame la marquise de Lambert, éd. revue et corrigée par Fontenelle, Amsterdam, 1766, p. 177.

10. Horace, Art poétique, trad. Jean-Jacques Porchat, Lyon, Louis Perrin, 1841, p. 32 , v. 309 : « La sagesse et le savoir, voilà la source de l'éloquence » (je traduis).

11. Daniel Auverlot, « Cicéron, ou le rêve d'une rhétorique idéale », dans Olivier Reboul et Jean-François Garcia (dir.), Rhétorique(s), Strasbourg, Presses universitaires de Strasbourg, 1989, p. 62. Voir également Alain Michel, Rhétorique et philosophie dans l'œuvre de Cicéron. Essai sur les fondements philosophiques de l'art de persuader, Paris, PUF, 1960, p. 80-149. 12. Cicéron, De l'orateur, Paris, Les Belles Lettres, 1971, liv. III, v, 19 : « [les choses et les mots] ne sauraient être séparés » («[res et verba] seiuncta esse non possunt »).

13. Ibid., liv. III, vi, 22 : « Una est enim [...] eloquentia ».

14. Ibid., xv, $56:$ : cogitandi pronuntiandique ratio». 
philosophie, du moins lorsque celle-ci se réclame de la tradition inaugurée par Socrate, car

ce fut lui [Socrate] qui [...] sépara l'art de bien penser et de bien dire [sapienterque sentiendi et ornate dicendi scientiam], deux sciences qui, au fond, tiennent l'une à l'autre. C'est d'alors que date cette séparation si importante entre, si j'ose dire, la langue et l'esprit [linguae atque cordis], divorce vraiment absurde, inutile et condamnable, qui fit en sorte que l'acquisition du savoir et de l'éloquence dût s'en remettre à deux maîtres différents [ut alii nos sapere, alii dicere docerent $]^{15}$.

Reconstituer l'unité perdue du dire et de la pensée constitue donc la tâche essentielle que s'était assignée Cicéron, et c'est justement à la lumière de ce premier souvenir lettré qu'il convient d'envisager la conception que se fait la marquise de Lambert de l'idéal auquel doivent aspirer ces « maisons où, écrivait-elle, il étoit permis de parler $\&$ de penser ».

Avec l'évocation de ces « maisons », il importe, en second lieu, de souligner combien la marquise de Lambert associe cet idéal rhétorique et philosophique hérité des Anciens à la tradition moderne du salon qui, elle, remonte au siècle précédent, tradition à laquelle elle demeure fortement attachée et qu'elle conçoit même comme menacée. C'est ce que montre à l'évidence cette petite histoire du salon que proposent ses Nouvelles réflexions sur les femmes en rappelant à quel point le Grand Siècle en fournit les modèles les plus achevés :

Madame Henriette d'Angleterre, qui auroit servi de modele aux Graces, donnoit l'exemple. Sous un visage riant, sous un air de jeunesse qui ne sembloit promettre que des jeux, elle cachoit un grand sens \& un esprit sérieux. Quand on traitoit, ou qu'on disputoit avec elle, elle oubloit son rang \& ne paraissoit élevée que par sa raison. Enfin l'on ne croyoit avancer dans l'agrément \& la perfection, qu'autant qu'on avoit su plaire à Madame. Un hôtel de Rambouillet, si honoré dans le siècle passé seroit le ridicule du nôtre. On sortoit de ces maisons comme des repas de Platon, dont l'ame étoit nourrie $\&$ fortifiée ${ }^{16}$.

À un siècle nouveau, indigne héritier des précédents illustres qui ont cessé de l'inspirer, s'oppose ainsi le salon classique, si digne, au contraire, des modèles antiques. Mais avec ces « grâces » que prodiguent des agréments qui ont pour vocation de conférer « un visage riant» à « un esprit sérieux », c'est surtout, on le pressent déjà, le rôle essentiel que jouent les femmes dans l'histoire du salon que ce texte revendique avec éclat. Posséder l'usage du monde, en sachant faire taire les distinctions de rang, et polir l'âpreté des disputes et des raisonnements, en recourant aux grâces d'une parole aimable : voilà, en somme, les principes

15. Ibid., xvi, 60-61.

16. Anne-Thérèse de Marguenat de Courcelles, marquise de Lambert, op. cit., p. 177-178. 
régissant la pratique du salon, dont la marquise de Lambert ne se fait l'historienne que pour mieux célébrer le magistère qu'exercent les femmes dans ces «maisons où, on s'en souvient, il étoit permis de parler \& de penser ».

Il résulte enfin de ces deux prémisses une configuration culturelle originale qu'anime et dynamise une alliance entre deux paradigmes : d'une part, on l'a vu, l'idéal cicéronien, à la fois antique et viril, d'une parole éloquente où s'exprime l'unité du dire et du savoir; et, d'autre part, l'apport du salon, essentiellement moderne, mondain et féminin. Lorsque la marquise de Lambert publie ses Nouvelles réflexions sur les femmes en 1727, on voit s'affirmer un peu partout cette même configuration, qui représente l'un des lieux communs du premier XVIII ${ }^{\mathrm{e}}$ siècle trop souvent méconnus et que la thèse récemment soutenue par Aurélie Zygel-Basso a fort bien mis en évidence ${ }^{17}$. Songeons, par exemple, à un roman comme celui que fait paraître la marquise de Lassay en 1726 et dont le seul titre, Tullie, fille de Cicéron ${ }^{18}$, annonce les aventures d'une jeune salonnière pleine d'esprit, laquelle prête les charmes de la conversation féminine aux préceptes professés par son père, incarnant ainsi à merveille les aspirations d'une modernité qui revisite l'idéal cicéronien au profit de la conversation mondaine. Mais se rêver en fille de Cicéron conviendrait tout autant à la marquise de Lambert, dont les Nouvelles réflexions sur les femmes entendent, précisément, faire triompher la conception d'une sociabilité lettrée où, suivant son expression, les Muses vivent « en société avec les Grâces ${ }^{19}$ ».

L'une des conséquences les plus fondamentales d'un tel dispositif social et culturel tient sans nul doute à la promotion d'un art de penser et de dire qui, désormais, est invité à s'accomplir dans un art de plaire. Cette transformation de la sensibilité, si caractéristique du premier $\mathrm{XVIII}^{\mathrm{e}}$ siècle, la marquise de Lambert la souligne à plusieurs reprises, observant même à quel point « on se lasse même d'admirer, si ce qu'on admire n'est aussi fait pour plaire ${ }^{20} »$. À cette évolution se rattache également la revalorisation des petits genres et, notamment, tous ces romans qui, comme l'écrit encore la marquise de Lambert, sont « faits par des Dames dont les ouvrages sont aussi aimables qu'elles ${ }^{21} »$. Surtout, la pratique de ces petits genres s'accompagne de l'invention d'un nouveau style, celui qu'illustre la prose de la marquise de Lambert elle-même et qui, en multipliant traits d'esprit et antithèses paradoxales, se veut alerte

17. Voir Aurélie Zygel-Basso, Le Conte de fées féminin au XVIII siècle, thèse de doctorat de $3^{\text {e }}$ cycle, lettres françaises, Université de Paris-Sorbonne / Paris IV, 2009.

18. Reine de Madaillan de Lesparre, marquise de Lassay, Histoire de Tullie, fille de Cicéron, par une dame illustre, Paris, Pierre Prault, 1726, p. 6.

19. Anne-Thérèse de Marguenat de Courcelles, marquise de Lambert, op. cit., p. 177.

20. Ibid., p. 189

21. Ibid., p. 171. 
et symétrisé, comme dans cette remarque écrite à propos de Madame de la Sablière : « [C]'étoit la plus séduisante personne du monde, \& [...] ses goûts, ou plutôt [s] es passions, se rendoient maîtres de son imagination $\&$ de sa raison; de maniere que ses goûts étoient toujours justifiés par sa raison $[\ldots]^{22}$. » Mais qu'il s'agisse de plaire, de composer un roman ou encore de symétriser ses phrases, chaque fois, on s'en aperçoit, le goût, la sensibilité et l'imagination sont appelés à se rendre maîtres de la raison et, en retour, à se voir justifiées par elle. C'est que, assure la marquise de Lambert, « rien n'est si absolu que la supériorité de l'esprit, qui vient de la sensibilité, \& de la force de l'imagination ${ }^{23}$ "), avant d'ajouter, plus loin : «Rien ne peut plaire à l'esprit qu'il n'ait passé par le cœur ${ }^{24}$. » En adoptant de semblables maximes, on ne saurait, en somme, envisager une raison privée des secours de l'imagination ou du goût, et cette conception éminemment oratoire d'une rationalité associée aux grâces de la parole féminine nous conduit enfin à la seconde question dont on a proposé l'examen.

\section{" Minerve à Cythère " ou comment la raison devient aimable}

La seconde grande conséquence qu'il faut tirer de cette prééminence d'un modèle de sociabilité lettrée qui se place sous le double patronage de Minerve et de Cythère concerne, en effet, la conception même de la raison. Cet enjeu, il revient peut-être à l'abbé Trublet de l'avoir le mieux résumé dans un texte de 1759 consacré à Fontenelle, alors qu'il rappelle combien, dans la prose de son siècle, « la raison [...] bannit le raisonnement, ou du moins l'appareil \& les longueurs de la Dialectique ", grâce aux charmes du « bel-esprit » s'unissant à « l'esprit philosophique ${ }^{25} »$. De même, comme le signalait déjà Gabriel Henri Gaillard dans son Essai de rhétorique françoise à l'usage des jeunes demoiselles, sans les ornements du discours, « on fuit » la raison, « on évite son redoutable aspect; [...] mais, quand la séduisante Élocution lui prête son puissant secours, [...] tout céde à ses charmes [...]. L'esprit toujours est la dupe du cour, \& le cœur est la dupe de l'aimable Élocution ${ }^{26} »$. Mais s'il est vrai, comme l'écrit encore Gaillard, que « la raison perd le droit de persuader quand elle n'est pas éloquente ${ }^{27} »$, cette dimension sensible, voire sensuelle de

22. Ibid., p. 184 .

23. Ibid., p. 185

24. Ibid., p. 208.

25. Nicolas-Charles-Joseph Trublet, Mémoires pour servir à l'histoire de la vie et des ouvrages de Mrs. de Fontenelle et de la Motte. Tirés du Mercure de France 1756, 1757 et 1758, et du Dictionnaire de Moreri édition de 1759, seconde édition, corrigée et augmentée, Amsterdam, Marc Michel Rey, 1759, p. 15 et p. 12; souligné dans le texte.

26. Gabriel Henri Gaillard, Rhétorique françoise, op. cit., p. 130.

27. Gabriel Henri Gaillard, « Lettre sur l'épopée françoise », Mélanges littéraires, Amsterdam, s. n., 1756, p. 149. 
l'intelligence se réclame, en même temps, d'une culture qui s'invente et se rêve en se recommandant de l'aimable autorité des agréments et des sentiments qu'enfante l'imagination. Qu'on en juge d'après ces remarques de la marquise de Lambert :

Or que ne doit-on pas aux agréments de l'imagination? C'est elle qui fait les Poëtes \& les Orateurs : rien ne plaît tant que ces imaginations vives, délicates, remplies d'idées riantes. Si vous joignez la force à l'agrément, elle domine, elle force l'ame \& l'entraîne; car nous cédons plus certainement à l'agrément qu'à la vérité. L'imagination est la source $\&$ la gardienne de nos plaisirs [...] toute l'ame est en elle ${ }^{28}$.

Dans ce passage, si l'âme tient tout entière à l'imagination et aux plaisirs délicats ou enjôleurs dont cette faculté est la source, c'est d'abord dans la mesure où, sur le plan philosophique, la marquise de Lambert fait sienne une conception dynamique de l'esprit humain, qu'elle envisage partout en termes d'activité et de capacité d'invention, avec ses corrélats inévitables d'attrait pour le changement et le mouvement des passions, la variété et la vivacité des idées ou des représentations. De fait, en parfait accord avec les thèses empiristes que les Modernes déduisent de la tradition épicurienne, la marquise de Lambert critique sans cesse l'illusion où nous sommes de pouvoir accéder à l'essence des choses, en refusant constamment de considérer les sensations qu'occasionnent les objets du monde extérieur comme la manifestation de qualités réelles qui leur appartiennent en propre. Autrement dit, bien loin d'exister hors de nous, ces qualités n'ont de réalité qu'en nous : elles se confondent, pour ainsi dire, avec tous les fantômes qu'enfante l'activité de notre imagination, alors que, sans y penser, nous transportons continuellement ces simulacres sur les objets qui nous sont extérieurs. Que les " qualités extérieures » ne puissent, par exemple, "être aimables par elles-mêmes », qu'elles ne le soient « que par les dispositions qu'elles trouvent en nous ${ }^{29} »$, la marquise de Lambert en veut d'ailleurs pour preuve « qu'un même objet ne fait pas les mêmes impressions sur tous les hommes; \& que souvent nos sentiments changent, sans qu'il y ait rien de changé dans l'objet ${ }^{30} »$. C'est que, conclut-elle surtout, les « qualités

28. Anne-Thérèse de Marguenat de Courcelles, marquise de Lambert, op. cit., p. 179-180.

29. Ibid., p. 188.

30. Ibid., p. 187. On pourra comparer ce passage à plusieurs développements conçus dans le même esprit chez Étienne Simon de Gamaches, qui rappelle ainsi, à propos des "graces $[\ldots]$ d'un beau visage », à quel point ses qualités «s'augmentent, ou $[\ldots]$ s'affoiblissent [...] selon les differentes dispositions où nous nous trouvons, sans qu'il arrive le moindre changement réel du côté de l'objet » ("Premier discours », Le système du cœur, ou Conjectures sur la manière dont naissent les différentes affections de l'âme, principalement par rapport aux objets sensibles, par Monsieur de Clarigny, Paris, Denys Dupuis, 1704, p. 25-27). Membre de l'Académie des sciences, Gamaches est, tout comme la marquise de Lambert, un proche de Fontenelle, son Système $d u$ 
agréables, qui ébranlent l'ame, \& qui donnent de si douces impressions, ne sont point réelles, ni propres à l'objet; elles se doivent à la disposition de nos organes, \& à la puissance de notre imagination ", tant et si bien que le cœur ne peut « dépendre des loix de la Justice», n'étant « soumis qu'à celles du plaisiri ${ }^{31} »$.

Par-delà la méfiance qu'avait témoignée Descartes ou Malebranche envers l'imagination, les considérations de la marquise de Lambert en faveur de cette faculté confèrent évidemment au sentiment une dignité philosophique nouvelle, elle-même favorable à une revalorisation de l'activité spontanée de la sensibilité humaine et de la puissance d'invention de l'esprit. Surtout, elles permettent d'unir l'exercice de la raison aux charmes de l'imagination au nom du caractère, exemplaire encore là, de l'intelligence féminine :

Ceux qui attaquent les femmes ont prétendu que l'action de l'esprit, qui consiste à considérer un objet, étoit bien moins parfaite dans les femmes, parce que le sentiment qui les domine, les distrait \& les entraîne. L'attention est nécessaire; elle fait naître la lumiere, pour ainsi dire, approche les idées de l'esprit \& les met à sa portée : mais chez les femmes, les idées s'offrent d'elles-mêmes \& s'arrangent plutôt par sentiment que par réflexion; la nature raisonne pour elles, \& leur épargne tous les frais. Je ne crois donc pas que le sentiment nuise à l'entendement; il fournit de nouveaux esprits, qui illuminent de maniere que les idées se présentent plus vives, plus nettes $\&$ plus démêlées ; \& pour preuve de ce que je dis, toutes les passions sont éloquentes. Nous allons aussi sûrement à la vérité par la force \& la chaleur des sentiments, que par l'étendue \& la justesse des raisonnements; \& nous arrivons toujours par eux plus vîte au but dont il s'agit, que par les connoissances. La persuasion du cœur est au-dessus de celle de l'esprit, puisque souvent notre conduite en dépend : c'est à notre imagination \& à notre cœur, que la nature a remis la conduite de nos actions, \& de ses mouvements ${ }^{32}$.

Chez les femmes, en somme, le sentiment préside à l'«action de l'esprit », mais de telle manière que le travail de la raison s'y confond avec les mouvements de la nature elle-même. « La nature raisonne pour elles », écrit ainsi la marquise de Lambert, et cette admirable formule

cœur s'ouvrant ainsi sur une dédicace où il célèbre l' « éloquence fine et délicate » de l'auteur des Entretiens sur la pluralité des mondes (ibid., « Epistre», n. p.). Sur cet auteur et cet ouvrage, on voudra bien se reporter à mon article, "Brillant" du style et réhabilitation du plaisir dans l'écriture moraliste d'Étienne Simon de Gamaches à Crébillon fils ", Cahiers de l'Association internationale des études françaises, $\mathrm{n}^{\circ} 59$, mai 2007, p. 159-173.

31. Anne-Thérèse de Marguenat de Courcelles, marquise de Lambert, op. cit., p. 187-188. À comparer, encore là, à ce qu'écrivait Gamaches, pour lequel le cœur est également « indépendant des loix de la justice, et $[. .$.$] ne peut être soumis qu'à celle de la nature »$ («Avertissement », Le système du cœur, op. cit., n. p.).

32. Ibid., p. 182-183. 
résume au mieux en quoi raison et imagination, vérité et sentiment, raisonnement et agréments forment ici un tout-ensemble appelé à servir de socle à cet idéal d'une « raison aimable » que le premier XVIII ${ }^{\mathrm{e}}$ siècle s'est tant plu à célébrer. Tantôt, la rapidité des opérations intellectuelles auxquelles préside la nature illumine les idées, tantôt la vivacité des associations qu'elle suggère conduit plus sûrement à la vérité, tantôt la moralité de notre conduite s'enracine dans un élan spontané d'identification à l'autre : chaque fois, enfin, la force des expressions qu'elle dicte détermine une conception de l'éloquence formée à l'école d'une parole féminine où se conjuguent justesse du raisonnement, chaleur du sentiment et bonheur de l'expression. C'est, au demeurant, à la lumière de ces principes que la marquise de Lambert définit le goût, qui est l'un des plus grands « avantages qu'on donne aux femmes ${ }^{33}$ » et qui consiste, précisément, à faire « appercevoir d'une maniere vive \& prompte, sans qu'il en coûte rien à la raison, tout ce qu'il y a à voir dans chaque chose ${ }^{34} »$.

Au cours des trente dernières années, et notamment dans le monde anglo-saxon, toute une part essentielle de la critique postmoderne des Lumières a fait siennes, on le sait, les thèses jadis développées par Max Horkeimer et Theodor W. Adorno dans La Dialectique de la raison ${ }^{35}$ (1947). Au Canada anglais par exemple, un ouvrage comme Les Bâtards de Voltaire, que John Ralston Saul faisait paraître en 1992, portait en soustitre cette mention : La Dictature de la raison en Occident ${ }^{36}$, se faisant ainsi l'écho des critiques qu'avait formulées l'École de Francfort et qui, pour l'essentiel, aperçoivent dans les Lumières l'origine d'une raison devenue pure mathématique, instrument par excellence d'une logique implacable, calculatrice et comptable, voire déshumanisée et totalitaire. Au rebours de cette lecture du XVIII ${ }^{\mathrm{e}}$ siècle, l'historien de la rhétorique doit, me semblet-il, rappeler le rôle central d'une conception de la rationalité qui reste profondément étrangère à une sorte de proto-positivisme desséchant et dont on voudra bien, au contraire, considérer l'originalité, voire la valeur paradigmatique au sein de la culture des Lumières. Adossée à une longue tradition rhétorique, cette raison aimable et ornée, relevée par les assaisonnements que lui confère la conversation féminine ne saurait, par

33. Anne-Thérèse de Marguenat de Courcelles, marquise de Lambert, op. cit., p. 180.

34. Ibid., p. 182.

35. Theodor W. Adorno et Max Horkeimer, La dialectique de la Raison. Fragments philosophiques, trad. Éliane Kaufholz, Paris, Gallimard, 1974 [1947].

36. John Ralston Saul, Les bâtards de Voltaire. La dictature de la raison en Occident, Paris, Payot, 1993 [1992]; sur la critique postmoderne des Lumières dans le monde anglo-saxon, voir Nicholas Hudson, "Are we "Voltaire's Bastards"? John Ralston Saul and Post-Modern Representations of the Enlightenment », Lumen, vol. XX, 2001, p. 111-121. 
sa nature même, devenir abstraction mathématique, et c'est ce qui fait assurément le prix de la théorie qu'en offrent les Nouvelles réflexions sur les femmes de la marquise de Lambert.

Marc André Bernier

Université du Québec à Trois-Rivières

Chaire de recherche du Canada en rhétorique 